\title{
LA COMPETENCIA SOCIOEMOCIONAL DOCENTE EN EL LOGRO DEL APRENDIZAJE DE LAS COMPETENCIAS GENÉRICAS DEL PERFIL DE EGRESO DE EDUCACIÓN MEDIA SUPERIOR
}

\author{
Patricia Garrido Natarén¹: Secretaría de Educación Pública. México \\ jolipatt@hotmail.com
}

Martha Leticia Gaeta González: Universidad Popular Autónoma del Estado de Puebla. México

Marthaleticia.gaeta@upaep.mx

\section{RESUMEN}

Los cambios en la estructura familiar, las dinámicas y crecientes exigencias sociales, las nuevas competencias exigidas a los docentes y los pobres resultados alcanzados en la Educación Media Superior del Sistema Educativo Mexicano (INEE, 2012), hace de la enseñanza un proceso complejo que incrementa las tensiones emocionales del profesor (Marchesi, 2008). De ahí que en este trabajo se busca indagar sobre el nivel de desarrollo de la competencia socioemocional docente que contribuya al logro del aprendizaje de las competencias genéricas del perfil de egreso de Educación Media Superior en México. Se utilizó una metodología mixta; en la fase cuantitativa se usaron dos instrumentos estandarizados: el Test de Inteligencia Emocional Mayer-SaloveyCaruso (MSCEIT) y el Maslach Burnout Inventory (MBI), los cuales se aplicaron a 172 profesores de educación media superior. En la fase cualitativa se entrevistó a cinco docentes con los niveles más altos y cinco con los más bajos de competencia socioemocional (según los resultados del MSCEIT).En el presente texto se dan a conocer los hallazgos de la indagación cuantitativa. Los resultados del estudio mostraron que, en general, los docentes poseen una habilidad suficiente que les permite un desenvolvimiento con cierto grado de éxito, es decir, capacidad de percibir emociones; de acceder, generar y utilizar las emociones para ayudar al pensamiento, y regular las emociones para que promuevan el crecimiento emocional e intelectual.

\footnotetext{
1 Autor correspondiente: Patricia Garrido Natarén: Secretaria de Educación Pública. México Correo: jolipatt@hotmail.com
} 
Asimismo, se encontraron parámetros no indicativos de diagnóstico de Síndrome de Burnout según el MBI.

PALABRAS CLAVE: Emociones - Competencias docentes - Competencia emocional Inteligencia emocional - Competencias genéricas - Formación integral - Perfil de egreso - Educación media superior - Síndrome de burnout

\title{
TEACHER'S SOCIO-EMOTIONAL COMPETENCY IN ACHIEVING GENERIC SKILLS OF THE GRADUATE PROFILEINHIGHER SECONDARY EDUCATION
}

\begin{abstract}
Changes in family structure, dynamics and growing social demands, the new skills required of teachers and the poor results achieved in the Higher Secondary Education of the Mexican Educational System (INEE, 2012), makes teaching a complex process that increases Teacher emotional tensions (Marchesi, 2008). Therefore, in this paper we seek to investigate the level of development of socio-emotional skills of teachers to contribute to the achievement of learning generic skills of the graduate profile of Secondary Education in Mexico. A mixed methodology was used; in the Quantitative Phase two standardized instruments were used: the Emotional Intelligence Test Mayer-Salovey-Caruso (MSCEIT) and the Maslach Burnout Inventory (MBI), which were applied to 172 secondary educationteachers. In the qualitative phase five teachers with the highest levels and five with the lowest level of socio-emotional competence were interviewed (by results of MSCEIT).In this paper we present the findings of the quantitative inquiry. The study results showed that, in general, teachers have a sufficient ability that allows them the development with some success, that is, the ability to perceive emotions; to access, generate and use emotions to help thinking, and regulate emotions to promote emotional and intellectual growth. Also, no diagnostic parameters indicative of burnout syndrome were found by means of MBI.
\end{abstract}

KEYWORDS: Emotions - Teaching skills - Emotional competence - Emotional intelligence - Generic skills - Comprehensive education - Graduate profile - Upper secondary education - Burnout

\section{INTRODUCCIÓN}


En los últimos años las estructuras sociales y familiares han experimentado diversos y acelerados cambios, por los que el sistema escolar ha sido objeto de crecientes exigencias y críticas sociales al intentar dar respuesta a los retos que se le plantean en la actual sociedad del conocimiento y la información. No obstante, la finalidad de la educación continua siendo el garantizar el desarrollo armónico de la personalidad de cada individuo y de su inserción social; en donde el docente -agente de la cultura- ha hecho frente al desafío y reconoce que la escuela es un espacio vital del que dispone la sociedad para asegurar la cultura y para formar ciudadanos. Consecuentemente, en las dos últimas décadas ha irrumpido con fuerza en la gestión educativa la palabra: competencia. Término globalizado al que la UNESCO (1999, citado por Argudín, 2010) define basándose en los "cuatro pilares de la educación", como el conjunto de comportamientos socio-afectivos y habilidades cognoscitivas, psicológicas, sensoriales y motoras que permiten llevar a cabo adecuadamente una actividad o una tarea.

De ahí que muchos de los propósitos de programas educativos actuales están definidos en razón del desarrollo integral centrado en competencias, en la búsqueda de la calidad educativa y de dar respuesta a las demandas sociales y profesionales actuales. Particularmente, en el nivel medio superior mexicano $(S E P, 2008)$ se busca desarrollar en los educandos competencias que les permitan comprender el mundo e influir en él, que les capacite para continuar aprendiendo de forma autónoma a lo largo de la vida, para desarrollar relaciones armónicas con quienes les rodean y participar eficazmente en su vida social, profesional y política (competencias genéricas). Para lograr esta propuesta se requiere que los docentes tengan las competencias profesionales necesarias para asumir un nuevo rol, que enfatiza su carácter de acompañante o facilitador del proceso, a partir de un dominio tal que les permita adaptarse a nuevas situaciones, despertar el interés del educando en el aprendizaje, una gestión de la clase en óptimas condiciones, logrando con ello aumentar las posibilidades de éxito en la tarea de enseñar.

En este contexto, no se trata sólo de transmitir conocimientos, sino además de trabajar con los alumnos (y consigo mismo) las competencias que contribuyan al desarrollo de aprendizajes autónomos, integrales, que preparen ciudadanos; incluyendo para ello además de las competencias cognitivas y procedimentales, las sociales y emocionales (Vaello, 2009).Así, los educadores deben ser más que transmisores de conocimientos, tienen que desempeñar papeles más complejos para desarrollar la creatividad, la curiosidad intelectual, las habilidades del pensamiento, salud física y emocional, el sentido de ciudadanía activa de los alumnos (competencias genéricas). Asimismo, deben conocer las claves para el incremento de la autoestima, el rendimiento académico y la visión de las posibilidades de aprendizaje presentes y futuras de sus estudiantes, mediante su compromiso, conocimientos y destrezas (Day, 2007).

En el Sistema Educativo mexicano se ha dispuesto que los profesores posean las competencias didácticas, pedagógicas y de contenido de sus asignaturas, necesarias para un desempeño adecuado, estableciéndose acciones de capacitación y/o actualización tendientes a su logro, con la consecuente mejora de la calidad educativa. No obstante, las acciones realizadas no han logrado impactar satisfactoriamente en la consecución de los objetivos educativos (INEE, 2012), tal y como lo demuestran los altos índices de reprobación y deserción de alumnos a nivel nacional (32.7\% y 14.9\% respectivamente) y los bajos niveles de eficiencia terminal (62.2\%) reportados en el ciclo escolar 2009-2010 en EMS (INEE, 2012). En virtud de sólo haber favorecido tanto en docentes como estudiantes el desarrollo de competencias cognitivas y procedimentales, en detrimento de dar importancia a la pluralidad de la naturaleza 
humana y su emotividad, condición necesaria para un desarrollo integral. Se ha olvidado además que en los procesos de enseñanza y aprendizaje intervienen seres altamente emotivos -profesor y alumnos- y que la enseñanza es una actividad intrínsecamente social (TALIS, 2009).

Tal y como los demuestran en sus trabajos diversos investigadores, que señalan la importancia de las emociones no sólo en los contenidos del pensamiento, sino también a los propios procesos implicados en éste como la toma de decisiones racionales y en las interacciones sociales (Bisquerra, 2009; Damasio, 1994; Chabot y Chabot, 2010;Palomera, Fernández-Berrocal y Brackett, 2008). De modo que, las emociones y las habilidades relacionadas con su manejo actúan sobre los procesos de aprendizaje, la salud mental y física, así como en el desempeño académico. Sin olvidar su importante función preventiva respecto a la aparición de múltiples trastornos en la población escolar como son: problemas de ansiedad y depresión, problemas de atención y razonamiento, impulsividad y bajo rendimiento escolar, agresividad y violencia, comportamientos conflictivos y antisociales.

Ante la presencia de las importantes evidencias positivas de las competencias socioemocionales en el contexto educativo, aportadas por las diversas investigaciones en el contexto nacional e internacional, y preocupados ante los problemas formativos presentes en la Educación Media Superior (EMS) de México, surgió la inquietud de indagar sobre el nivel de desarrollo de estas competencias en los profesores que puedan contribuir al aprendizaje de las competencias genéricas de EMS. Asimismo, advertir alguna relación entre el nivel de competencia socioemocional docente y el desarrollo del Síndrome de Burnout docente.

\section{METODOLOGÍA}

\subsection{Participantes}

Para el desarrollo de este estudio, la población docente $(n=301)$ de cuatro planteles de Educación Media Superior en una ciudad al sur-este de México, fue sometida a un proceso de selección probabilística aleatoria estratificada, de la cual se integró una muestra de 172 docentes, 80 hombres y 90 mujeres, de entre 23 y 71 años de edad.

\subsection{Instrumentos}

Test de Inteligencia Emocional Mayer-Salovey-Caruso (MSCEIT, 2009), adaptación Española por Extremera y Fernández-Berrocal. Instrumento que pretende medir la aptitud para identificar, facilitar, comprender y manejar las emociones (141 ítems). Se trata de una escala de capacidad, es decir, mide cómo ejecutan las tareas las personas y resuelven problemas emocionales; la capacidad de razonar utilizando las emociones y

la capacidad de utilizar los sentimientos para mejorar el pensamiento. La inteligencia emocional evaluada con el MSCEIT implica cuatro componentes o ramas referidos a las siguientes capacidades: percepción emocional, facilitación emocional, manejo emocional y comprensión emocional. El análisis de los datos se realizó de acuerdo con la siguiente guía (ver Tabla 1).

Tabla 1. Guía para la interpretación de puntuaciones del MSCEIT 


\begin{tabular}{|c|c|c|}
\hline Rango de CIE & Rango cualitativo & Descripción \\
\hline Menor de 70 & Necesita mejorar & $\begin{array}{l}\text { Es posible que exista } \\
\text { cierta dificultad en esta } \\
\text { área, podría ser útil } \\
\text { mejorar las habilidades } \\
\text { en ella. }\end{array}$ \\
\hline Entre 70 y 89 & Puede mejorar & $\begin{array}{l}\text { Esta área no constituye } \\
\text { un punto fuerte, por lo } \\
\text { que puede ser interesante } \\
\text { tratar de mejorarla. }\end{array}$ \\
\hline Entre 90 y 110 & Competente & $\begin{array}{l}\text { La persona posee una } \\
\text { habilidad suficiente en } \\
\text { esta área que el permite } \\
\text { desenvolverse con cierto } \\
\text { grado de éxito. }\end{array}$ \\
\hline Entre 111 y 130 & Muy competente & $\begin{array}{l}\text { Esta área constituye un } \\
\text { punto fuerte para la } \\
\text { persona. }\end{array}$ \\
\hline Mayor de 130 & Experto & $\begin{array}{l}\text { Área muy desarrollada, } \\
\text { con elevado potencial en } \\
\text { ella. }\end{array}$ \\
\hline
\end{tabular}

Fuente: Mayer, Salovey y Caruso (2009)

Maslach Burnout Inventory (MBI, 1996). Instrumento que consta de 22 ítems que se distribuyen en tres escalas denominadas: realización personal en el trabajo (8 items), agotamiento emocional (9 items) y despersonalización (5 items). El análisis de los datos se realizó según la metodología propuesta por Maslach, la cual establece que puntuaciones altas en las dimensiones de cansancio emocional (CE) y despersonalización (DP) y bajas en realización personal (RP) diagnostican a la persona con el síndrome (ver Tabla 2).

Tabla 2. Puntuaciones establecidas para el MBI.

\begin{tabular}{l|lll}
\hline Burnout & $C E$ & $D P$ & $R P$ \\
\hline Bajo & 18 o menos & 5 o menos & 33 o menos \\
Medio & 19 a 26 & 6 a 9 & 34 a 39 \\
alto & 27 o más & 10 o más & 40 o más \\
\hline
\end{tabular}

Fuente: Maslach, Jackson y Leiter (1996).

\subsection{Procedimiento}

La aplicación de los instrumentos a los docentes se llevó a cabo en el propio centro escolar, de manera grupal. Las instrucciones fueron dadas de manera oral al inicio de la aplicación por uno de los investigadores del estudio. Se aseguró la confidencialidad de los datos y se reiteró a los participantes el que pudieran realizar preguntas en cualquier momento. Los datos recolectados a partir del MBI y del MSCEIT fueron 
procesados en el Sofware estadístico SPSS 20 (Statistical Package for Social Sciences) para su análisis.

\section{$2.4 \quad$ RESULTADOS}

\subsection{Características socio-demográficas de la muestra}

En relación a las características sociodemográficas de la muestra, podemos mencionar que la antigüedad de los docentes en el ejercicio profesional se encuentra entre los 2 años y los 43 años (Gráfico 1). Los perfiles profesionales de los profesores fueron muy diversos (ingeniería, profesional docente, psicología, profesional del área de la salud, derecho, profesional administrativo, profesional del área computacional, otras profesiones), encontrándose el mayor porcentaje de profesionistas (29.4\%) en el área de ingeniería, seguida por el área administrativa en un $22.4 \%$. Cabe resaltar que sólo el $11.2 \%$ de los educadores cuentan con estudios profesionales en docencia (Gráfico 2). Finalmente en cuanto al nivel educativo de los educadores, encontramos que el $43 \%$ tiene nivel de licenciatura, el $42 \%$ ha realizado una maestría y tan sólo el $2 \%$ tiene estudios de doctorado.

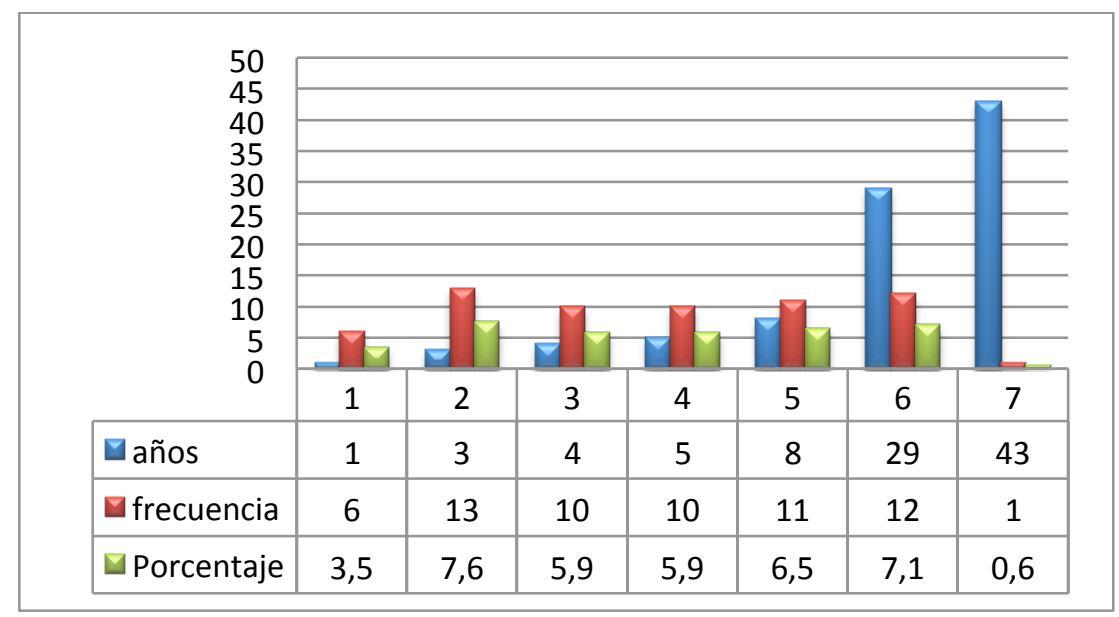

Gráfico 1. Antigüedad en el ejercicio docente

Fuente: elaboración propia.

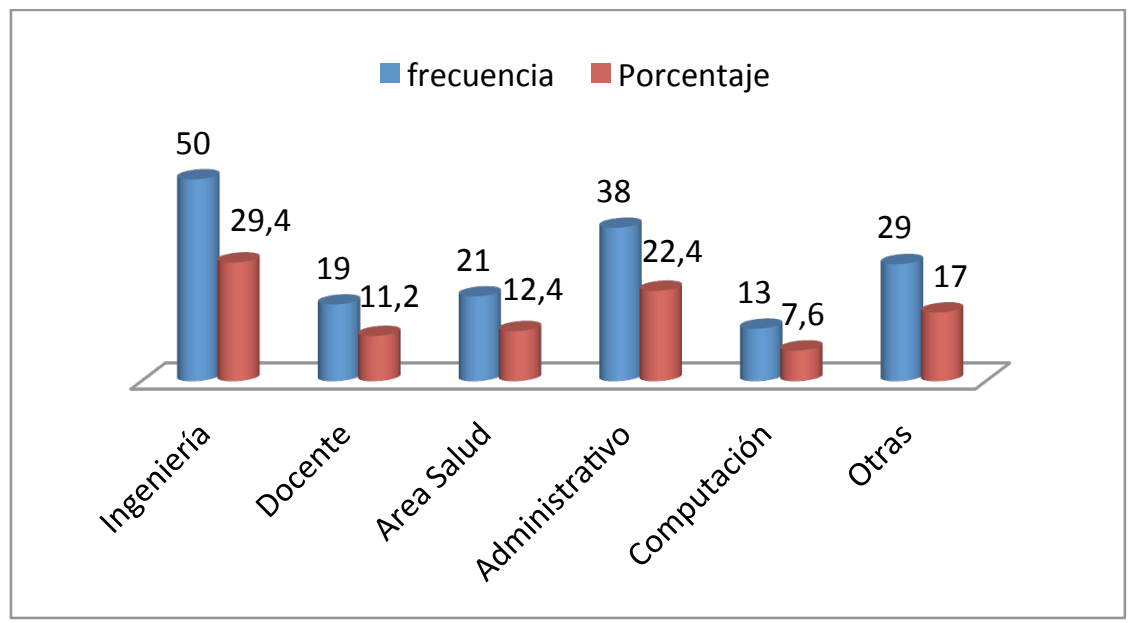

Gráfico 2. Perfil profesional docente 
Fuente: elaboración propia.

\subsection{Competencia socioemocional docente}

Para los fines de este trabajo únicamente se consideraron las puntuaciones totales del MSCEIT (CIE), las dos puntuaciones de área (Experiencial CIEX y Estratégica CIES), y las cuatro puntuaciones de rama (Percepción emocional CIEP, Facilitación emocional CIEF, Comprensión emocional CIEC, y Manejo emocional CIEM).

Con respecto a las puntuaciones de rama, creímos importante resaltar los valores obtenidos respecto de la percepción emocional, en virtud de mostrar un decremento en cuanto a su valor máximo (131) respecto las otras tres puntuaciones (135), que repercute en el valor de la media (98.82) y en la desviación típica (12.027). A continuación presentamos los resultados generales globales encontrados (ver Tabla 3):

Tabla 3. Puntuaciones docentes del MSCEIT

\begin{tabular}{lcccc}
\hline \multicolumn{1}{c}{$\mathrm{N}=170$} & Mínimo & Máximo & Media & Desv. típ. \\
\hline Inteligencia Emocional & 65 & 135 & 103.21 & 13.35 \\
$\begin{array}{l}\text { Inteligencia Emocional } \\
\text { Estratégica }\end{array}$ & 65 & 135 & 105.57 & 13.18 \\
Facilitación Emocional & 65 & 135 & 102.91 & 14.01 \\
Manejo Emocional & 69 & 135 & 104.98 & 12.90 \\
Percepción Emocional & 65 & 131 & 98.82 & 12.03 \\
Comprensión Emocional & 65 & 135 & 104.35 & 13.90 \\
Inteligencia Emocional & 65 & 135 & 100.59 & 12.92 \\
Experiencial & & & & \\
\hline
\end{tabular}

Fuente: elaboración propia

En el gráfico 3, podemos apreciar los resultados generales globales encontrados, en donde sólo cuatro docentes fueron identificados con competencias socioemocionales de expertos (valores de 130 y más en la escala valorativa) que representan el $2.35 \%$ de la muestra de estudio. El 51.17\% de los docentes fueron reportados como competentes, en virtud de encontrarse entre los valores de 90 y 109 en la escala. Finalmente, para 24 profesores se emitió la recomendación de desarrollar y/o mejorar dichas competencias ya que presentaron valores inferiores a 89 dentro de la escala valorativa, representando el $14.11 \%$ del total de la muestra. 


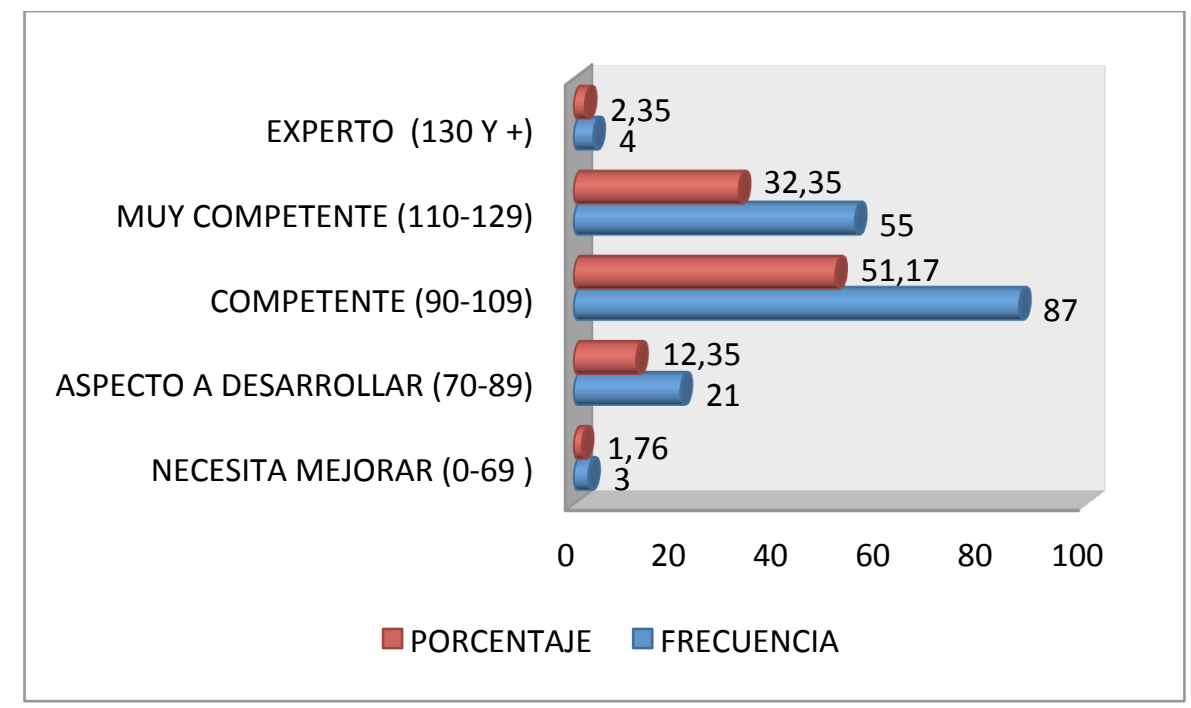

Gráfico 3. Niveles de competencia socioemocional docente

Fuente: elaboración propia

En relación al género, encontramos que los niveles de desarrollo de la competencia socioemocional fueron ligeramente más altos en las mujeres que en los hombres, ya que el $54.44 \%$ de ellas presentó valores por encima de la media, mientras que en ellos sólo el $41.25 \%$ registro valores de competencia por encima de la media total (103.21 CIE).

Tabla 4. Desarrollo de competencia socioemocional docente por género

\begin{tabular}{lcccc}
\hline Género & \multicolumn{2}{l}{$>$ de $\mathbf{1 0 3 . 2 1}$} & \multicolumn{2}{c}{$<$ de $\mathbf{1 0 3 . 2 1}$} \\
\hline Hombre & 33 & $(41.25 \%)$ & 47 & $(58.75 \%)$ \\
Mujer & 49 & $(54.44 \%)$ & 41 & $(45.55 \%)$ \\
\hline
\end{tabular}

Fuente: elaboración propia

\subsection{Síndrome de Burnout}

Se realizó el análisis de los datos para cada uno de los sujetos de la muestra, considerando la metodología propuesta por Maslach et al. (1986), a fin de identificar las puntuaciones correspondientes para cada dimensión y la confiablidad de los datos recabados. En la Tabla 5 podemos apreciar las frecuencias según las dimensiones.

Tabla 5. Frecuencias por dimensión del MBI

\begin{tabular}{cccc}
\hline Dimensión & Nivel Alto & Nivel Intermedio & Nivel Bajo \\
\hline Agotamiento emocional & 5 & 8 & 157 \\
Despersonalización & 9 & 32 & 129
\end{tabular}


Fuente: elaboración propia

Respecto de la distinción de indicadores del Síndrome de Burnout en los docentes, como un elemento orientador para un diagnóstico de su estado emocional, podemos mencionar que encontramos un agotamiento emocional bajo (92.35\%), despersonalización baja (75.88\%) y alta realización personal $(78.82 \%)$, lo cual según los parámetros establecidos en el MBI no son indicativos de diagnóstico de Síndrome de Burnout.

Por tanto, con el propósito de poder determinar si los docentes presentaban algún nivel o fase de desarrollo del Síndrome de Burnout, se consideró el modelo de Golembiewsky, Munzenraider y Carter (1983, citado por Lozano et al., 2008). Lográndose de esta manera establecer las frecuencias de incidencia por niveles de afectación (Gráfico 4), en donde la mayor frecuencia se localizó en el nivel III, con bajo Burnout, correspondiendo al $60.58 \%$, y la menor frecuencia en el nivel $\mathrm{V}$ de intermedio Burnout $(1.17 \%)$.

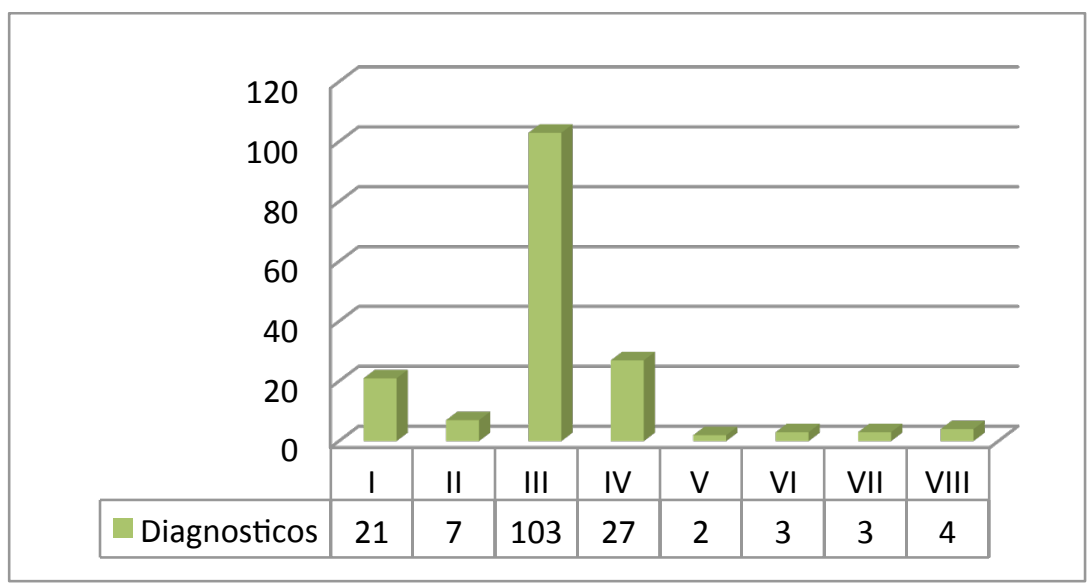

Gráfico 4. Nivel de Burnout (Golembiewski et al., 1983).

Fuente: elaboración propia

En cuanto a la evaluación del Síndrome de Burnout, considerando las puntuaciones de todos los niveles, se encontró que la mayoría de los docentes (77.05\%) presentaron bajos niveles de desarrollo y sólo diez docentes (5.89\%) evidenciaron puntuaciones indicativas de niveles altos del Síndrome (ver Gráfico 5). 


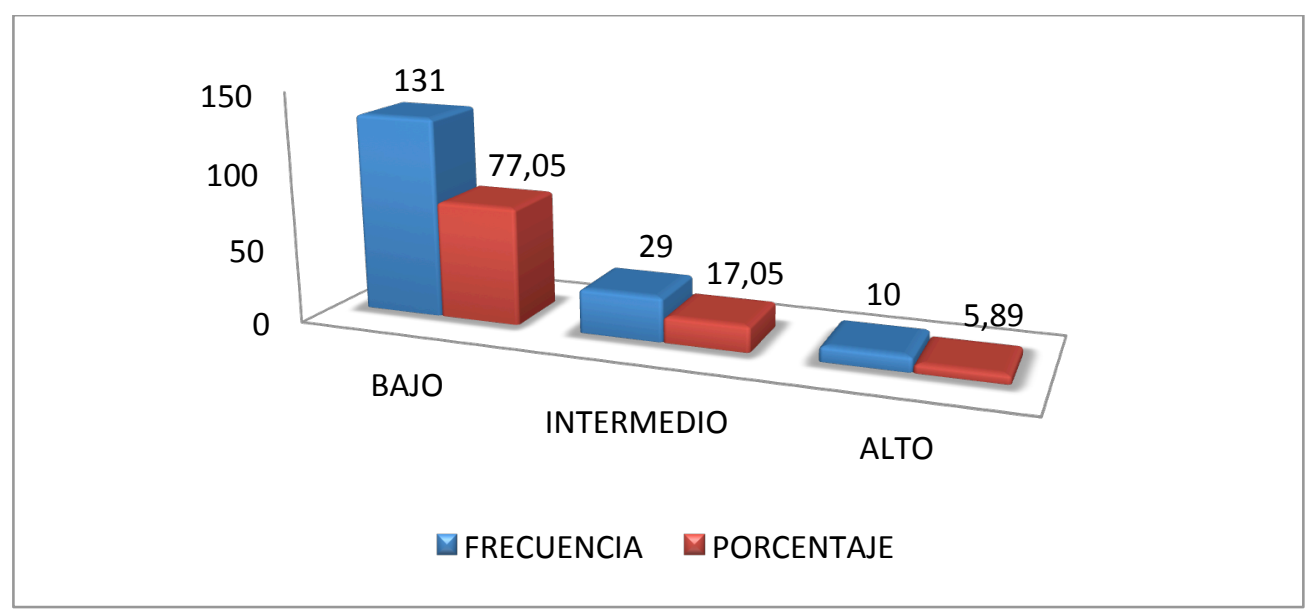

Gráfico 5. Niveles de Síndrome de Burnout docente

Fuente: elaboración propia

Al relacionar los niveles del Burnout detectados con el sexo de los docentes encuestados, podemos apreciar que los hombres evidenciaron en un $76.25 \% \quad(n=61)$ niveles bajos del Síndrome, mientras que la proporción en las mujeres con estos mismos niveles fue de $77.77 \%(n=70)$. Los niveles altos fueron mostrados en los hombres en 6 casos y en las mujeres en 4. Asimismo, al vincular los años de antigüedad docente con los niveles de Burnout encontrados, llama la atención que presenta una mayor incidencia el Síndrome en los primeros años de ejercicio en 33 profesores (en el rango de 3 a 5 años de antigüedad).

Con el propósito de detectar algún beneficio preventivo de las competencias socioemocionales en el desarrollo del Síndrome de Burnout, tal y como lo sugieren algunos autores (Doménech, 2010; Extremera y Duran, 2007; Extremera y FernándezBerrocal, 2005), decidimos comparar los niveles de S. de Burnout docente identificados según el modelo de Golembiewsky et al. (1983), con los niveles de competencia socioemocional docente, determinados por el MSCEIT (Tabla 6). Observándose en el presente estudio que este beneficio preventivo no se hace evidente en los resultados comparativos de la prueba tangencial que determina que dichas variables son independientes una de la otra. Consideramos que lo anterior puede deberse a que resulta difícil atribuir a una causa al Síndrome de Burnout y sus consecuencias, ya que es ingente la cantidad de factores que ocurren durante la práctica docente para la aparición del estrés laboral.

Tabla 6. Relación entre niveles de C. Socioemocional y S. de Burnout.

\begin{tabular}{|c|c|c|}
\hline Nivel de Competencia IE & Frecuencia & Nivel Síndrome Burnout \\
\hline Experto (130 y más) & 4 & 3 de Nivel Bajo \\
1 de Nivel Intermedio & $\begin{array}{c}44 \text { de Nivel Bajo } \\
10 \text { de Nivel Intermedio } \\
1 \text { de Nivel Alto }\end{array}$ \\
\hline Muy competente (110-129) & 55 & \\
\hline
\end{tabular}




\begin{tabular}{|c|c|c|}
\hline Competente (90-109) & 87 & $\begin{array}{c}64 \text { de Nivel Bajo } \\
14 \text { de Nivel Intermedio } \\
9 \text { de Nivel Alto }\end{array}$ \\
& & 18 de Nivel Bajo \\
& & 3 de Nivel Intermedio \\
\hline Aspecto a desarrollar (70-89) & 21 & 2 de Nivel Bajo \\
& & 1 de Nivel Intermedio \\
\hline
\end{tabular}

Fuente: elaboración propia

\section{ANÁLISIS Y DISCUSIÓN}

Con base en los resultados encontrados podemos concluir, respecto al grado de desarrollo de las competencias socioemocionales docentes en los sujetos de la muestra, que su nivel de competencia (según la escala valorativa del MSCEIT) es indicativo de poseer una habilidad suficiente que permite un desenvolvimiento con cierto grado de éxito (media=103.21), es decir, una capacidad de percibir emociones; de acceder, generar y utilizar las emociones para ayudar al pensamiento, y regular las emociones para que promuevan el crecimiento emocional e intelectual.

No obstante lo anterior, consideramos importante señalar que la percepción emocional fue la dimensión menos desarrollada (media de 98.82). Asimismo, los diez reportes con niveles de competencia más bajos se identificaron en docentes con edades entre 50 y 68 años. De igual forma, se detectó en las 24 puntuaciones más bajas de competencia socioemocional (según los resultados del MSCEIT), que diez corresponden a profesores con un rango de antigüedad en el ejercicio docente de 21 a 30 años, y ocho a maestros con un rango de 4 a 10 años de antigüedad. Es por ello que consideramos necesario profundizar sobre los aspectos condicionantes en futuras investigaciones.

Estos resultados sugieren que los docentes con bajos niveles de competencia socioemocional realizan un gran esfuerzo para regular no sólo sus propias emociones, sino también la de sus alumnos; siendo para ellos un desafío el ajustar el currículo a la heterogeneidad de sus alumnos, el despliegue de habilidades para establecer relaciones interpersonales, el crear entornos favorables de aprendizaje, la toma de decisiones, etc. (Extremera y Fernández-Berrocal, 2005; Palomera, Fernández-Berrocal y Brackett, 2008; Waisburg, 2008). Lo anterior, en virtud de la gran importancia que las emociones tienen en el proceso educativo al guiar la atención, que a su vez dirige el aprendizaje y la memoria (Damasio, 1994, 2010; Day, 2007; Jensen, 2010); al hacer que los contenidos sean personalmente interesantes y profundamente sentidos, aspecto esencial para una buena enseñanza (Day, 2007). De igual forma, estos profesores presentan menor capacidad de prevenir y superar el estrés, y por lo tanto mayor riesgo de desarrollar el Síndrome de Burnout, lo que pone en peligro su bienestar docente y su desempeño en el aula (Lazarus y Folkman, 1984; Lazarus, 1993; Mattews y Zeidner, 2000; Zaidner y Endler, 1996; citados por Bisquerra, 2009). 
Por su parte, Martínez-Otero (2008) afirma que al enseñar el profesor no solamente comunica conocimientos, sino que muestra su personalidad, comunica con su postura, tono de voz, mirada, gestos, etc., creando con ello un clima de aula que puede ser positivo para el aprendizaje o por el contrario negativo, dificultando el logro de los propósitos educativos. En este mismo sentido, Maturana (2001) afirma que las emociones constituyen el aspecto de mayor relevancia para facilitar los aprendizajes en educación, ya que emociones positivas y ambientes gratos permitirán la realización de acciones favorables para el aprendizaje, y emociones negativas o ambientes no gratos las obstaculizarán. Por tanto, la complejidad relacional de la clase demanda una adecuada preparación del docente para interpretar y organizar el aula, realizando una acertada negociación con los alumnos y estableciendo el conjunto de tareas y contenidos más adecuados para su formación (Tobón, et al., 2010), aspectos en los que una competencia socioemocional docente, una adecuada formación didácticapedagógica y un profundo compromiso ético, pueden ayudar al profesor en el logro de las metas educativas.

En relación a los resultados encontrados de la aplicación del MBI podemos concluir que la causa de desarrollo del Síndrome de Burnout y sus consecuencias, es ingente debido a la diversidad de factores presentes durante la práctica docente para la aparición del estrés laboral. Tal y como lo señala el modelo teórico explicativo del Síndrome de Burnout desarrollado por Gil-Monte y Peiró (1997, citado por Gil-Monte, 2001) basado en la teoría estructural, la cual plantea que el estrés es consecuencia de falta de equilibrio de la percepción entre las demandas y la capacidad de respuesta del sujeto, siendo importantes en su desarrollo factores como los antecedentes personales. Como ejemplo de ellos, podemos mencionar a la experiencia, la autoestima, las características de la personalidad, etc.; interpersonales vinculados a la relación educativa (desmotivación del estudiante, mala relación con sus pares, escasa disciplina, etc.); y a factores organizacionales, como son la sobrecarga laboral, escasez de recursos, etc. Dado que las habilidades de inteligencia emocional (competencia socioemocional) se encuentran dentro de los antecedentes personales de los factores relacionados, entonces habría que indagar en futuros estudios qué nivel de influencia tienen los factores organizacionales y los vinculados a la relación educativa en los docentes de EMS en el desarrollo de niveles altos e intermedios de Síndrome de Burnout en esta población de estudio. Consideramos importante reiterar que esta afección no es sólo una amenaza para la salud física y psíquica docente, sino que tiene importantes repercusiones en sus relaciones interpersonales (con alumnos, pares, padres de familia, etc.), dañando significativamente su ejecución profesional y la calidad educativa, al presentar ausentismo, abandono, etc., tal y como lo refieren diversos autores (Belcastro, 1982; Calvete y Villa, 2000; Chakravorty, 1989; Gibbs, 2001; Landsman, 2001; Maslach, 1992; Matud, Garcia y Matud, 2002; Quevedo-Aguado et al., 1999; Smith, 2001; Söderfeldt, Söderfeldt y Warg, 1995, citados por Moriana y Herruzo, 2004).

\section{BIBLIOGRAFÍA}

ARGUDÍN, Y. (2010). Educación basada en competencias. Nociones y antecedentes. México: Trillas. 
BISQUERRA, A. R. (2009). Psicopedagogía de las emociones. España: Síntesis.

CANO, E. (2010). Cómo mejorar las competencias de los docentes. Guía para la autoevaluación y el desarrollo de las competencias del profesorado. Barcelona: Graó.

CARMONA, G.M. (2008). Hacia una formación docente reflexiva y crítica: fundamentos filosóficos. Venezuela. Recuperado de: http:/ / redalyc.org/articulo.oa?id $=65216719007$

CHABOT, D. \&CHABOT, M. (2010) Pedagogía emocional. Sentir para aprender. México: Alfaomega.

DAMASIO, A. (1994). El error de descartes. España: Drakontos

DAMASIO, A (2010). Y el cerebro creó al hombre. España: Destino.

DAY, CH. (2007). Pasión por enseñar. La identidad personal y profesional del docente y sus valores. España: Narcea.

DELORS, J. (1996). La educación encierra un tesoro. Francia: UNESCO/Santillana.

DOMÉNECH, B. F. (2010). Análisis de los estilos de pensamiento que utilizan los profesores españoles en el aula. España. Recuperado de: http://www.revistaeducacion.mec.es/doi/358_088.pdf

ESTUDIO INTERNACIONAL SOBRE DOCENCIA Y APRENDIZAJE (2009). Creación de entornos eficaces para la enseñanza y el aprendizaje: nota informativa sobre México. OCDE. Recuperado de: http://168.255.201.80/talis2009/Nota_pais_TALIS.pdf

EXTREMERA N. \& DURÁN, A (2007). Inteligencia emocional y su relación con los niveles de burnout, engagement y estrés en estudiantes universitarios. España. Recuperado de: http:/ / saber.ucv.ve/xmlui/bitstream/123456789/273/1/Lectura\%20\%20INTELI 1.PDF

EXTREMERA, N. \& FERNÁNDEZ-BERROCAL, P. (2005). La importancia de desarrollar la inteligencia emocional en el profesorado. España. Recuperado de: http://www.rieoei.org/deloslectores/759Extremera.PDF

FERNÁNDEZ-BERROCAL, P. \& EXTREMERA, N. (2005). La inteligencia emocional y la educación de las emociones desde el Modelo de Mayer y Salovey. España. Recuperado de:http:/ / www.redalyc.org/articulo.oa?id=27411927005

FERNÁNDEZ-BERROCAL, P. \& RUÍZ, A. D. (2008). La inteligencia emocional en la educación. España. Recuperado de: http://www.investigacionpsicopedagogica.org/revista/new/ContadorArticulo.php?256 
GIL-MONTE, P.R. (2001). El síndrome de quemarse por el trabajo (Síndrome de Burnout): aproximaciones teóricas para su explicación y recomendaciones para la intervención.España. Recuperado de: http://psicopediahoy.com/burnout-teoriaintervencion/

INSTITUTO NACIONAL PARA LA EVALUACIÓN EDUCATIVA. (2011). Panorama Educativo de México. México. Recuperado de: http://www.inee.edu.mx

INSTITUTO NACIONAL PARA LA EVALUACIÓN EDUCATIVA. (2012). Panorama Educativo de México. Indicadores del Sistema Educativo Nacional. México. Recuperado http:/ / publicaciones.inee.edu.mx/buscadorPub/P1/B/111/P1B111.pdf

JENSEN, E. (2010). Cerebro y aprendizaje. Competencias e implicaciones educativas. España: Narcea.

LOZANO, L.M., CAÑADAS, G., MARTIN, M., PEDROSA, I., SUAREZ, J. \& COLS. (2008). Descripción de los niveles de burnout en diferentes colectivos profesionales. España. Recuperado de: http:/ / dialnet.unirioja.es/servlet/articulo?codigo $=2856102$

MARCHESI, A. (2008).Sobre el bienestar de los docentes. Competencias, emociones y valores. España: Alianza.

MARTÍNEZ-OTERO, V. (2008). El discurso Educativo. Madrid: Editorial CCS.

MASLACH, C.; JACKSON, S.E. \& LEITER, M.P. (1996). Maslach Burnout Inventory, Third Edition. EE. UU: Consulting Psychologist Press.

MATURANA, H. (2001). Emociones y lenguaje en educación y política. Chile: Dolmen.

MAYER, J.D.; SALOVEY, P. \& CARUSO, D.R. (2009).Test de inteligencia emocional Mayer-Salovey-Caruso MSCEIT. España: TEA ediciones.

MORIANA, E.J. Y HERRUZO, C.J. (2004). Estrés y burnout en profesores. España. Recuperado de: http:/ / www.redalyc.org/articulo.oa?id=33740309

PALOMERA, M.R.; GIL-OLARTE, M.P. \& BRACKETT, M. A. (2006).¿Se perciben con inteligencia emocional los docentes? Posibles consecuencias sobre la calidad educativa. España.

de:http:/ / dialnet.unirioja.es/servlet/articulo?codigo=2165292

Recuperado

PALOMERA, R.; FERNÁNDEZ-BERROCAL P. \&BRACKETT, M.A. (2008). La inteligencia emocional como una competencia básica en la formación inicial de los docentes: algunas evidencias. España/EE.UU. Recuperado de: www.investigacionpsicopedagogica.org/revista/new/ContadorArticulo.php?276 
PALOMERO, F. P. (2009). Desarrollo de la competencia social y emocional del profesorado: aproximación desde la psicología humanista. España. Recuperado de: http://www.redalyc.org/pdf/2170/217015206011.pdf

SECRETARIA DE EDUCACIÓN PÚBLICA. (2008). Acuerdo Secretarial No. 442 por el que se establece el Sistema Nacional de Bachillerato en un marco de diversidad. Diario Oficial de la Federación. México. Recuperado de: http://www.cosdac.sems.gob.mx/riems.php

TOBÓN, T. S., PIMIENTA, P. J. \& GARCÍA, F. J. (2010). Secuencias Didácticas: aprendizaje y evaluación de competencias. México: Pearson.

VAELLO, O. J. (2007). Cómo dar clases a los que no quieren. Madrid: Santillana.

VAELLO, O. J. (2009). El profesor emocionalmente competente. Un puente sobre aulas turbulentas. España: Graó.

VAILLANT, D. \&ROSSEI, C. (2006). Maestros en Latinoamérica hacia una radiografía de la profesión. Santiago de Chile: PREAL.

WAISBURG, C. G. (2008). La emoción en la toma de decisiones ¿perjuicio o beneficio? Argentina. Recuperado: de http://www.tandemsd.com/newsletter/marzo/La_emoción_en_la_toma_de_decisio nes_-_por_CW.pdf

\section{Patricia Garrido Natarén}

Docente investigador de tiempo completo de Educación Media Superior en el Estado de Veracruz. Sus intereses de indagación comprenden la formación y desarrollo de competencias docentes; la inteligencia emocional y las competencias genéricas del perfil de egreso de EMS. Ha realizado diversas acciones de supervisión y asesoría a profesores y participando en diversos programas de actualización docente.

\section{Martha Leticia Gaeta González}

Profesora investigadora de tiempo completo en el Doctorado en Pedagogía de la Universidad Popular Autónoma del Estado de Puebla, México. Sus principales intereses de investigación se centran en la autorregulación del aprendizaje en diferentes niveles educativos, desde un marco socio-cognitivo. En esta área ha publicado libros, capítulos de libro y diversos artículos en revistas nacionales e internacionales de alto impacto y ha impartido comunicaciones en diferentes foros. Es miembro del Sistema Nacional de Investigadores (SNI) en México. 
Diciembre 2016 Año XIX (137) pp. 108-123 